\title{
The effects of the carboxyl-terminus amino acids of the Shiga toxin B-subunit on retrograde transport
}

\author{
DAN LIU, YUYING FAN, JIE LI, XIAOGE GAO, MIAO HAO, HUITING XUE and GUIHUA TAI \\ School of Life Sciences, Northeast Normal University, Changchun, Jilin 130024, P.R. China
}

Received February 20, 2012; Accepted April 2, 2012

DOI: $10.3892 / \mathrm{mmr} .2012 .885$

\begin{abstract}
The Shiga toxin B-subunit (STxB), from the enteric pathogen, Shigella dysenteriae, is responsible for the attachment of its receptor, globotriaosylceramide (Gb3), and navigates the retrograde pathway from the plasma membrane to the endoplasmic reticulum (ER). In this study, in order to demonstrate the role of carboxyl-terminus (C-terminus/al) amino acids of the B-fragment on the retrograde transport speed and the retrograde transport pathway, STxB was modified by site-directed mutagenesis and by the addition of an amino acid tail. The results showed that when the C-terminal amino acid, arginine [Arg (R)], was mutated to serine [Ser (S)], the speed of the B-fragment transportation into the ER at $37^{\circ} \mathrm{C}$ was slower. When an acidic amino acid tail 'glutamine (Glu)-Ser' (ES) was added to the C-terminal amino acid 'R', the B-fragment transporting speed slowed down and remained in the Golgi apparatus. Further experiments showed that the effects induced by mutations of the amino acid tail resulted in $\mathrm{STxB}-\mathrm{EEEES} \geq$-EEES $>$-EES $>$-ES, demonstrating that the retardation effect on the tail was increased and the length of the acidic amino acid was augmented. The effect was possibly produced by an acidic amino acid tail, not only by the amino acid ' $E$ '. The significant inhibitory effect on the speed of B-fragment retrograde transport was observed only when the mutations of the acidic amino acid tail were linked near to the C-terminus. These results may provide important insights for the study of transport mechanisms and for the development of STXB serial proteins as vectors for drug delivery.
\end{abstract}

\section{Introduction}

The Shiga toxin (STx) is a cytotoxic protein secreted by the enteric pathogen, Shigella dysenteriae, which causes hundreds of millions of people to be infected with severe dysentery

Correspondence to: Dr Yuying Fan or Dr Guihua Tai, School of Life Sciences, Northeast Normal University, 5268 Renmin Street, Changchun, Jilin 130024, P.R. China

E-mail: fanyy033@nenu.edu.cn

E-mail: taigh477@nenu.edu.cn

Key words: Shiga toxin B-fragment, retrograde transport, carboxylterminus, site-directed mutagenesis annually worldwide (1). STx is composed of one A-subunit (STxA) and five identical B-subunits (STxB). STxB ( 8 kDa protein) is capable of binding to the toxin receptor, globotriaosylceramide (Gb3) anchored in the host cell membrane via a trisaccharide moiety (Gal $\alpha$ (1-4)-Gal $\beta$ (1-4)-Glc) (2). STXB may assemble in a ring-like pentameric structure in a non-covalent manner, which carries the A subunit into the cell (3). To attack the cytosol of the host cell, the STx navigates the retrograde pathway sequentially through the plasma membrane, early endosome/recycling endosome (EE/RE) and the Golgi apparatus to the endoplasmic reticulum (ER) (4). Once STx reaches the ER, one fragment of STxA releases and translocates to the cytosol, which can modify $28 \mathrm{~S}$ rRNA, and cause cell death through the inhibition of protein synthesis (5).

The retrograde transport route used by the STx family has received considerable attention, not only in microbiology but also in cell biology. STxB provides valuable information on endogenous pathways of intracellular transport, which may aid in the investigation of endosome transport and sorting mechanism(6-8), and supply novel methods for investigating subcellular organelle characteristics. For instance, STxB can be used to measure the $\mathrm{pH}$ of the ER at rest and during calcium release (9). Recombinant STxB has been exploited in immunotherapy (10). Moreover, Gb3 is expressed at markedly higher levels in colon carcinoma cells and in certain other cancers $(11,12)$; therefore it was hoped that it could be used as a receptor to achieve drug delivery $(13,14)$. For instance, STxB has been developed as an intracellular delivery tool for cancer imaging (15), targeted chemotherapy (16) and immunotherapy $(17,18)$. Bouter et al even attempted to carry functionalized spherulites into cells (19). Therefore, STxB has broad prospects to be exploited in cancer therapy and immunotherapy.

Certain related toxins, such as Cholera toxin and Pseudomonas exotoxin A, have KDEL sequences, an ER retention signal, which could help to transfer molecules to the ER by COPI-coated vesicles. However, neither Ricin nor STx have a KDEL sequence and they are nevertheless transported from the Golgi apparatus to the ER (20-22). Girod et al found that STxB is transported through a COPI-independent (21) and Rab 6-dependent transport route from the Golgi to the ER $(6,7)$; however, how STxB transports from the Golgi to the ER remains unclear.

The carboxyl terminus (C-terminus/al) of the B-fragment sticks out of the globular structure of the protein. After STxB binds to its receptor, Gb3, the C-terminal of STxB stretches 
out the side of the cell membrane, so the modification of the C-terminus will not destroy STxB structure and inhibit its binding activity directly. Consequently, the C-terminus of STxB has been used to connect with one signal peptide (4), carrying biomarkers (23) and exogenous drugs. Modification, such as the addition of an N-glycosylation site and KDEL peptide, has not been shown to affect the transport path of the B subunit (4). However, the role of the C-terminus in the transport of STxB remains clear.

In the present study, we reconstructed a series of B-subunit proteins by site-directed mutagenesis and the addition of an amino acid tail at the C-terminus of STxB, and compared the effects of all the mutants on STXB retrograde transport pathways. The results may provide new opportunities for STXB to be applied to cancer treatment as a vector for drug delivery.

\section{Materials and methods}

Reagents and antibodies. RPMI-1640 medium was purchased from Invitrogen (Carlsbad, CA, USA). Fetal bovine serum, penicillin and streptomycin were purchased from PAA (PAA Laboratories GmbH, Pasching, Austria). Trypsin and PMSF were from Amersco. Mouse monoclonal anti-STxB antibody $13 \mathrm{C} 4$ was prepared as described previously $(4,8)$. HeLa and 13C4 cells were from American Type Culture Collection (Manassas, VA, USA). FITC-conjugated goat anti-mouse IgG was purchased from Proteintech Group Inc. (Chicago, IL, USA). Monoclonal antibody against human Golgin-97 was from Molecular Probes (Eugene, OR, USA). All restriction enzymes and calf intestinal alkaline phosphatase were purchased from Takara (Shiga, Japan). Pfu DNA polymerase and dNTPS were purchased from Promega (Madison, WI, USA).

Preparation of recombinant STXB and mutation (STXB-X). PSTxB-GLy-KDEL plasmid [a generous gift from Dr Ludger Johannes (Institut Curie/CNRS, Paris, France)] containing the full sequence of STXB was used as the PCR template for the preparation of the recombinant STxB and its mutation. The forward primers, Shiga AtpE, and the reverse primers, STB-X, were used (Table I). The PCR product was digested by NotI and $S p h \mathrm{I}$, and inserted into the pSTxB(sulf)2 between the Not $\mathrm{I}$ and $S p h I$ sites (referred to as pSTxB-X). The DNA sequences were verified by dideoxy-sequencing (Shanghai Sangon Biological Engineering Technology and Services Co., Ltd., Shanghai, China).

The plasmid, pSTB-X, expressing recombinant STxB was transformed into Escherichia coli $(E$. coli) DH5 $\alpha$ cells, then extracted and purified as previously described (24). After incubation overnight at $30^{\circ} \mathrm{C}$, the cells were incubated at $42^{\circ} \mathrm{C}$ for $4 \mathrm{~h}$. The periplasm protein was prepared by osmosis shock, then purified by affinity chromatography with Q-Sepharose Fast Flow and Mono Q Column.

Cell cultures. HeLa cells were maintained in RPMI-1640 medium supplemented with $10 \%$ (v/v) heat-inactivated fetal bovine serum, $100 \mu \mathrm{g} / \mathrm{ml}$ penicillin and streptomycin and $10 \mathrm{mmol} / 1 \mathrm{HEPES}$ at $37^{\circ} \mathrm{C}$ in a $5 \% \mathrm{CO}_{2}$ atmosphere.

STXB retrograde transport assay. HeLa cells were grown on $12-\mathrm{mm}$ round glass cover slips at a density of $1.0 \times 10^{5}$ cells/

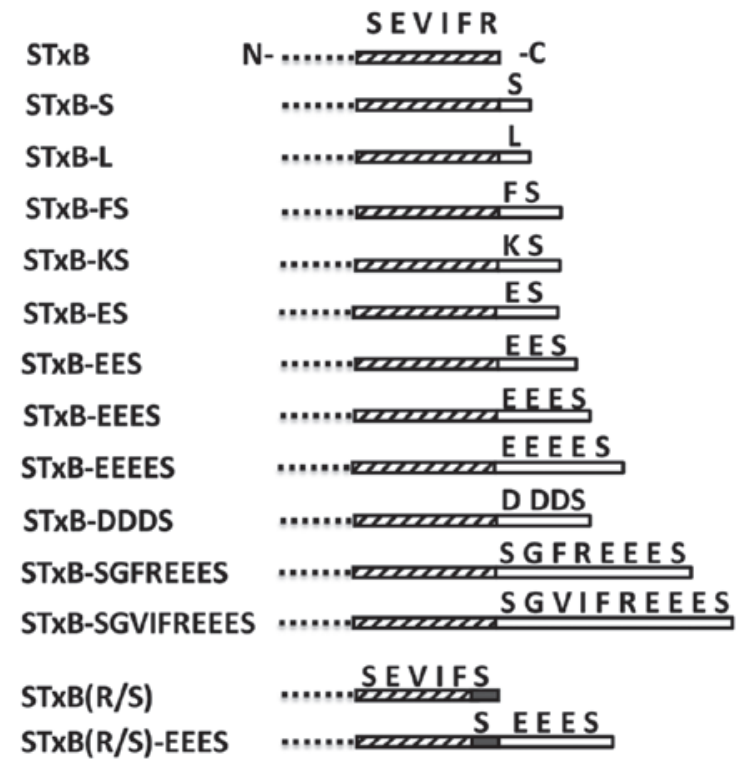

Figure 1. The structures at the carboxyl-terminus of the Shiga toxin B-subunit (STXB) and mutants.

well. After $40 \mathrm{~h}, 0.5 \mu \mathrm{M}$ of STxB and various mutants were internalized into cells at $17.5^{\circ} \mathrm{C}$ for $1.5 \mathrm{~h}$ to synchronize transport from the endosome to Golgi at EE/RE. The medium was then removed and replaced with fresh medium. Cells were incubated at $37^{\circ} \mathrm{C}$ for $3 \mathrm{~h}$. After washing with PBS/CM (PBS containing $1 \mu \mathrm{M} \mathrm{CaCl}_{2}, 1 \mu \mathrm{M} \mathrm{MgCl}$ ), the cells were fixed in paraformaldehyde for $30 \mathrm{~min}$. Cells were washed with $\mathrm{NH}_{4} \mathrm{Cl}$ / PBSCM and PBS/CM alternatively, incubated in $0.1 \%$ Triton $\mathrm{X}-100 / \mathrm{PBS} / \mathrm{CM}$ and blocked in dilution buffer. Subsequently, cells were incubated with the first antibody for $1 \mathrm{~h}$ and washed with PBS/CM. Cells were then incubated with the second antibody for $1 \mathrm{~h}$ and washed. Finally, cells were mounted and examined under a fluorescence microscope.

\section{Results}

Preparation of a series of aberrant STXB. To demonstrate the contribution of amino acids at the C-terminus of STxB to retrograde transport, a series of mutants were reconstructed by site-directed mutagenesis and the addition of an amino acid tail, and their retrograde pathways were also compared by immunofluorescence assay. Therefore, after designing primers and constructing plasmids, all the proteins were expressed in $E$. coli DH5 $\alpha$ cells, and purified by Q-Sepharose Fast Flow and Mono Q Column. The purity and specificity were measured by SDS-PAGE and western blot analysis, and the results suggested that the mutants could bind to the original receptor, 13C4 (data not shown). The structure features of all the mutants are shown in Fig. 1. Gene sequencing results showed that the mutants were correctly expressed (Table II).

The effect of $C$-terminal amino acids on the retrograde transport of $S T x B$. STxB and various mutants were incubated with cells at $37^{\circ} \mathrm{C}$ for $3 \mathrm{~h}$ and the location of the protein was observed by immunofluorescence assay. The results showed that any modification of the amino acid at the C-terminal did not change the pathway of the B-subunit trafficking, which still 
Table I. PCR primers.

Primer sequences (5'-3')

\begin{tabular}{ll}
\hline Shiga AtpE & CACTACTACGTTTTAAC \\
STxB & TATATTAGAGGCCTCAACGAAAAATAACTT \\
B(R/S $)$ & TATATTAGCGGCCGCTTCATGAAAAAATAACTTCGCTGAA \\
B-L & TATATTAGCGGCCGCTTCAAAGACGAAAAATAACTTCGC \\
B-S & TATATTAGCGGCCGCTTCATGAACGAAAAATAACTTCGC \\
B-KS & TATATTAGCGGCCGCTTCATGATTTACGAAAAATAACTTCGC \\
B-FS & TATATTAGCGGCCGCTTCATGAAAAACGAAAAATAACTTCGC \\
B-ES & TATATTAGCGGCCGCTTCATGATTCACGAAAAATAACTTCGC \\
B-EES & TATATTAGCGGCCGCTTCATGATTCTTCACGAAAAATAACTTCGC \\
B-EEES & ATTAGCGGCCGCTTCATGATTCTTCTTCACGAAAAATAACTTCGCT \\
B-EEEES & TATATTAGCGGCCGCTTCATGATTCTTCTTCTTCACGAAAAATAACTTC \\
B-DDDS & ATTAGCGGCCGCTTCATGAATCATCATCACGAAAAATAACTTCGCT \\
B(R/S)-EEES & ATTAGCGGCCGCTTCATGATTCTTCTTCTGAAAAAATAACTTCGCTGAA \\
B-SGFR-EEES & TAGAGCGGCCGCTTCATGATTCTTCTTCACGAAATCCAGAACGAAAAATAACTTC \\
B-SGVIFR-EEES & \\
\hline
\end{tabular}

STxB, Shiga toxin B-subunit.

Table II. Sequence alignment of Shiga toxin B-subunit (STxB) and fusion proteins.

\begin{tabular}{ll} 
Sequences & \\
\hline STxB & GCGAAGTTAT TTTTCGTTGA GCGGCCGCTA TCATCCTAAT TCTACTAGCT \\
STxB(R/S) & GCGAAGTTAT TTTTTCATGA AGCGGCCGCT AATGACTCAG AATAGCTCAG \\
STxB-L & GCGAAGTTAT TTTTCGTCTT TGAAGCGGCC GCTAATGACT CAGAATAGCT \\
STxB-S & GCGAAGTTAT TTTTCGTCA TGAAGCGGCC GCTAATGACT CAGAATAGCT \\
STxB-FS & GCGAAGTTAT TTTTCGTTT TCATGAAGCG GCCGCTAATG ACTCAGAATA \\
STxB-KS & GCGAAGTTAT TTTTCGTAAA TCATGAAGCG GCCGCTAATG ACTCAGAATA \\
STxB-ES & GCGAAGTTAT TTTTCGTGAA TCATGAAGCG GCCGCTAATG ACTCAGAATA \\
STxB-EES & GCGAAGTTAT TTTTCGTGAA GAATCATGAA GCGGCCGCTA ATGACTCAGA \\
STxB-EEES & GCGAAGTTAT TTTTCGTGAA GAAGAATCAT GAAGCGGCCG CTAATGACTC \\
STxB-EEEES & GCGAAGTTAT TTTTCGTGAA GAAGAAGAAT CATGAAGCGG CCGCTAATGA \\
STxB-DDDS & GCGAAGTTAT TTTTCGTGAT GATGATTCAT GAAGCGGCCG CTAATGACTC \\
STxB(R/S)EEES & GCGAAGTTAT TTTTTCAGAA GAAGAATCAT GAAGCGGCCG CTAATGACTC \\
STxB-SGFREEES & GCGAAGTTAT TTTTCGTTCT GGATTTCGTG AAGAAGAATC ATGAAGCGGC \\
STxB-SGVIFEEES & GCGAAGTTAT TTTTCGTTCT GGAGTTATTT TTCGTGAAGAAGAATCATGA
\end{tabular}

Underlined sequences indicate site-directed mutagenesis and the addition of amino acid tails; bold sequences indicate C-terminal residues of STxB.

followed the EE/RE-Golgi-ER route. However, the mutations with differently charged amino acids with the same end serine [Ser (S)] affected the trafficking efficiency of the B-fragments (-ES, -LS, -FS, -KS). Modification with -ES slightly reduced the transport efficiency. Further experiments showed that another acidic amino acid, aspartic acid [Asp (D)], also had the same activity, indicating that the effects were not specific to glutamine [Glu (E)]. This reduction of efficiency was cumulative by increasing the number of acidic amino acids. Additionally, it was found that the acidic amino acid tail (-EEES) must be close to the end of the B-subunit, otherwise, the effect of the acidic amino acid tail was decreased or even lost. According to the results of the mutants of STxB retrograde transport, it was confirmed that the C-terminal of STxB plays an important role in its intracellular transport.

The effects of the charge properties of amino acids. HeLa cells were incubated with $0.5 \mu \mathrm{M}$ of STxB or $\mathrm{STxB}(\mathrm{R} / \mathrm{S})$ at $17.5^{\circ} \mathrm{C}$ for 


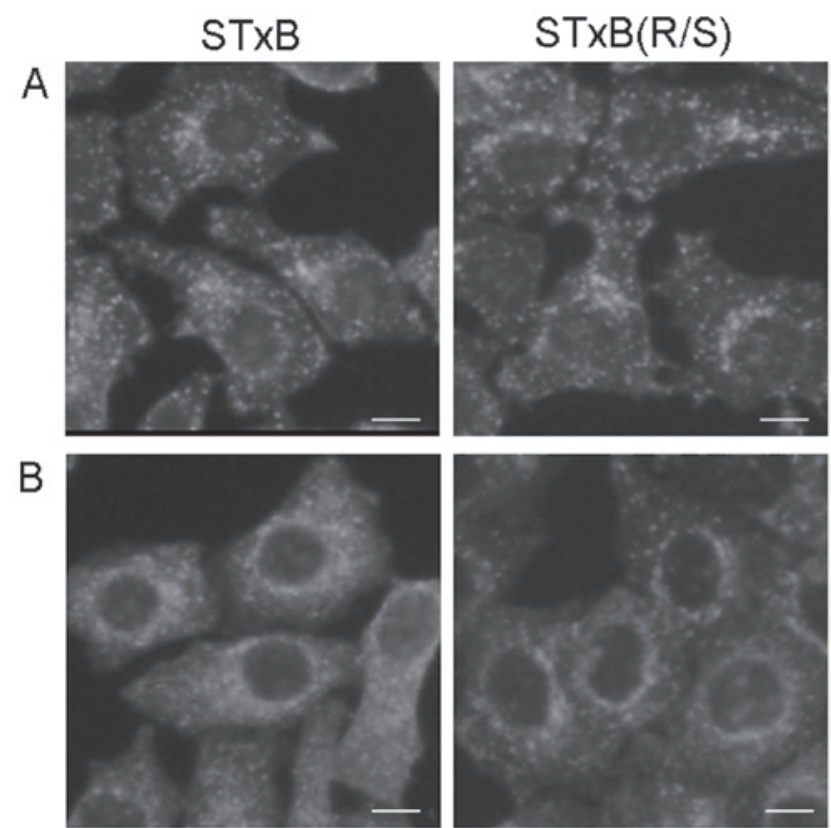

Figure 2. Internalization of the Shiga toxin B-subunit $(\mathrm{STxB})$ and $\mathrm{STxB}(\mathrm{R} / \mathrm{S})$ into HeLa cells. Adherent HeLa cells were incubated at (A) $17.5^{\circ} \mathrm{C}$ for $1.5 \mathrm{~h}$, and then (B) $37^{\circ} \mathrm{C}$ for $3 \mathrm{~h}$ with $0.5 \mu \mathrm{M} \mathrm{STxB}$ and $\mathrm{STxB}(\mathrm{R} / \mathrm{S})$. Cells were fixed and immunolabeled with antibodies against the toxin, and their retrograde transport was compared under a fluorescence microscope. Scale bar, $10 \mu \mathrm{M}$.

$1.5 \mathrm{~h}$. The B fragments were immunolabeled with specific antibody. The results showed that $\mathrm{STxB}(\mathrm{R} / \mathrm{S})$ and $\mathrm{STxB}$ have almost the same efficiency during transport from the plasma membrane to the $\mathrm{EE} / \mathrm{RE}$, and the majority of the dots remained at $\mathrm{EE} / \mathrm{RE}$ (Fig. 2A). However, when the cells were cultured for another $3 \mathrm{~h}$, the transport efficiency of $\mathrm{STxB}(\mathrm{R} / \mathrm{S})$ was lower than $\mathrm{STxB}$ on the trafficking pathway EE/RE-Golgi-ER. It was observed that $\mathrm{STxB}(\mathrm{R} / \mathrm{S})$ and $\mathrm{STxB}$ transported from the endosomes to the Golgi apparatus, with a number of $\mathrm{STx}(\mathrm{R} / \mathrm{S})$ markedly aggregating into the crescent-like Golgi apparatus near the nucleus in cells, while STxB transported into the ER (Fig. 2B).

Taken together, these results suggest that $\mathrm{STxB}(\mathrm{R} / \mathrm{S})$ transports from the plasma membrane to the endosome at the same speed as $\mathrm{STxB}$, and $\mathrm{STxB}(\mathrm{R} / \mathrm{S})$ aggregates into the Golgi apparatus more evidently than STxB. Therefore, an alkaline amino acid residue at the $\mathrm{C}$-terminus of STxB may promote the effective transport from the Golgi apparatus to the ER.

Effect of the addition of an amino acid tail to the C-terminus. The alkaline amino acid ' $R$ ' at the C-terminus may play a positive role in the transport of STxB from the Golgi to ER. Therefore, we aimed to study the effects of the addition of an amino acid tail with a different charge to the alkaline amino acid ' $\mathrm{R}$ ' on retrograde transport. A series of mutants were prepared with an amino acid ' $S$ ' at the end, which could keep the expression and stability. They were named STxB-S, STxB-L and STxB-ES (adjoined with an acidic amino acid), STxB-FS (adjoined with a neutral amino acid), STxB-KS (adjoined with a basic amino acid).

HeLa cells were incubated with $0.5 \mu \mathrm{M}$ of STxB or its mutants at $17.5^{\circ} \mathrm{C}$ for $1.5 \mathrm{~h}$, and continued to be cultured at $37^{\circ} \mathrm{C}$ for another $3 \mathrm{~h}$. Cells were fixed and immunolabeled with STxB antibody. When the cells were incubated with STxB, STxB-S, STxB-L, STxB-FS and STxB-KS, there were many diffusion dots through the cytoplasm, indicating that the majority of protein trafficked into the ER. However, some dots aggregated into a crescent-like positioning with a few diffusion dots in the cell cytoplasm treated with STxB-ES, indicating that there were more STxB-ES residing in the Golgi apparatus but not in the ER (Fig. 3). Additionally, STxB-L
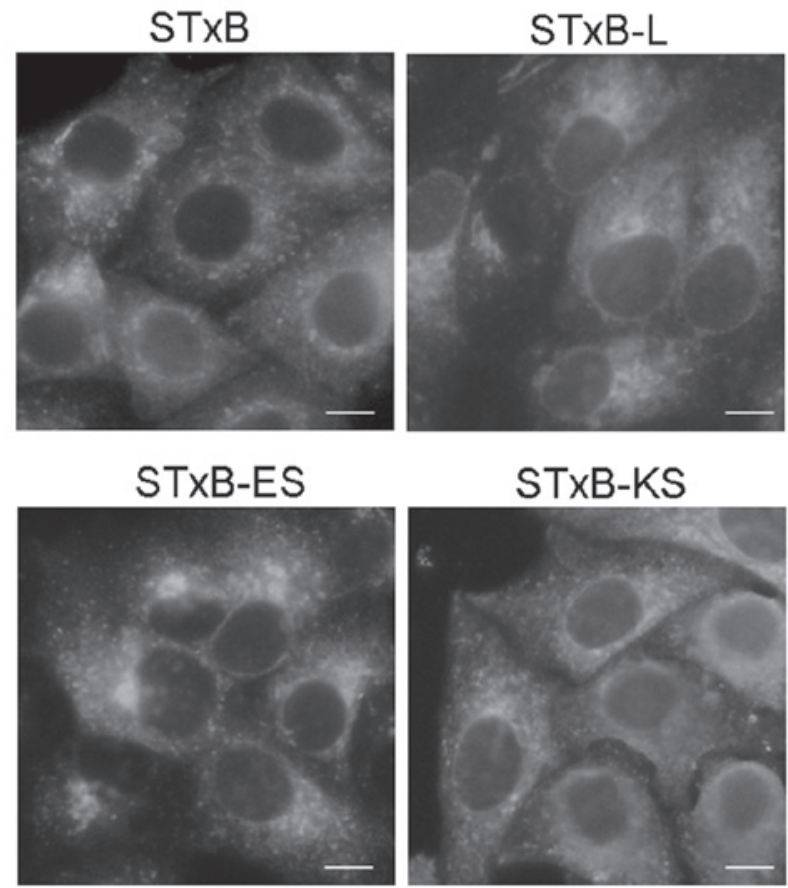

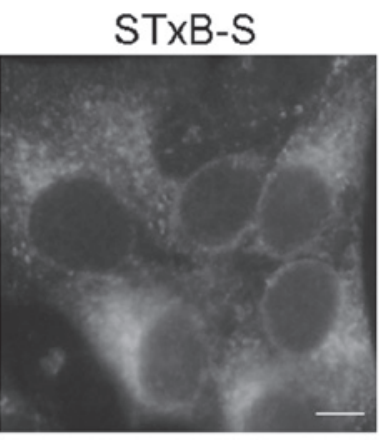

STXB-FS

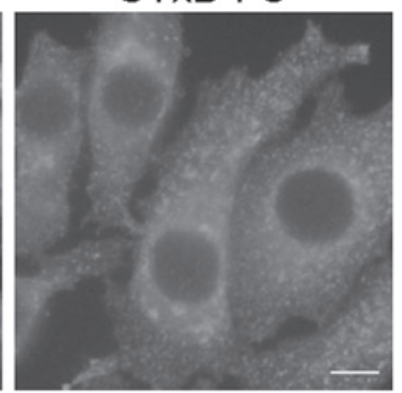

Figure 3. Effects of the addition of an amino acid tail at the carboxyl-terminus on retrograde transport in HeLa cells. Scale bar, $10 \mu \mathrm{M}$. STxB, Shiga toxin B-subunit. 

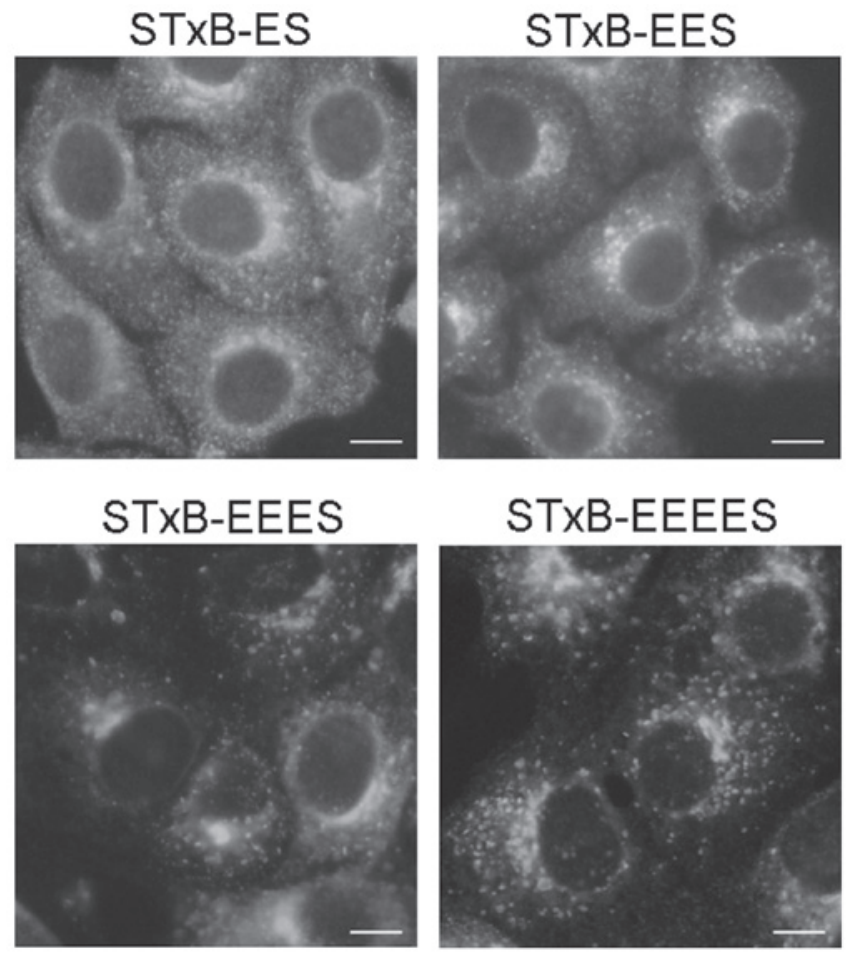

Figure 4. Effects of various lengths of the acidic amino acid tail at the carboxyl-terminus on retrograde transport in HeLa cells. Scale bar, $10 \mu \mathrm{M}$. STxB, Shiga toxin B-subunit.

showed a similar effect with STxB-S, indicating that the effect is not caused by the terminal amino acid ' $\mathrm{S}$ '.

Above all, the results reveal that the addition of an amino acid tail to the C-terminus of STxB can affect its transport speed from the Golgi to the ER; the acidic amino acid 'ES' adjoined to the C-terminus of STxB can slow down its transport speed from the Golgi to the ER.

Effect of the number of acidic amino acids. Adding an 'ES' tail to the C-terminus of STxB resulted in B-fragments remaining in the Golgi apparatus; therefore, we further investigated the effect of the number of acidic amino acid 'E's on STxB retrograde transport.

$\mathrm{HeLa}$ cells were incubated at $17.5^{\circ} \mathrm{C}$ for $1.5 \mathrm{~h}$ and $37^{\circ} \mathrm{C}$ for another $3 \mathrm{~h}$ with $0.5 \mu \mathrm{M}$ of STxB-ES, -EES, -EEES and
-EEEES. The immunofluorescence assay that probed for STxB demonstrated that the length of the 'ES' tail affected the B-fragment trafficking speed. Transport speed from the Golgi to the ER was slowed down more notably when we increased the amino acid number from 'ES' to 'EEEES'. This means that there will be an accumulation of B-fragments remaining in the Golgi apparatus (Fig. 4). The extent of the reduction in the speed of STxB transport from the Golgi to the ER is as follows: STxB-EEEES $\geq$-EEES>-EES>-ES.

Effect of the specificity of the acidic amino acid. The amino acid tail with the acidic amino acid ' $E$ ' adjoined to the C-terminus of STxB can significantly slow down B-fragment transport from the Golgi to the ER, but it is unknown whether this effect is specific to ' $E$ '.

To solve this problem, the mutants, STxB-EEES and -DDDS, were reconstructed and their transport speeds were compared following the method mentioned above. It was found that B-EEES and -DDDS have the same effect of slowing down transport and presenting an evident Golgi apparatus position compared to the prototype (Fig. 5). Accordingly, the effective slowing down of toxin protein transport may be caused by the modification of the $\mathrm{C}$-terminus with acidic amino acids, which was not 'E' specific.

Effect of the position of the acidic amino acid tail. When the acidic amino acid tail was added close to the $\mathrm{C}$-terminus of STxB, B-fragment transport from Golgi apparatus to the ER was slowed. In order to determine what effect the position of the tail at the C-terminus has on B-fragment retrograde transport, trafficking pathways of the mutants $\mathrm{STxB}(\mathrm{R} / \mathrm{S})$-EEES, -SGFREEES and -SGVIFR-EEES, which have different lengths of amino acid fragments inserted between the acidic amino acid tail and the C-terminus, were studied and compared. Cells treated with $\mathrm{B}(\mathrm{R} / \mathrm{S})$-EEES caused $\mathrm{B}$-fragments to be transported at a low speed from the Golgi to the ER and the majority of them were observed near the Golgi apparatus. However, a higher amount of protein arrived at the ER in the cells treated with B-SGFR-EEES and B-SGVIFR-EEES as well as STxB after the same amount of time (Fig. 6). Therefore, the position at which the acidic amino acid tail is added is important for the retrograde transport of STxB. When the end amino acid ' $R$ ' was mutated to 'S' and then added with an amino acid tail 'EEES' to be $\mathrm{STxB}(\mathrm{R} / \mathrm{S})$-EEES, the slowed transport effect was most
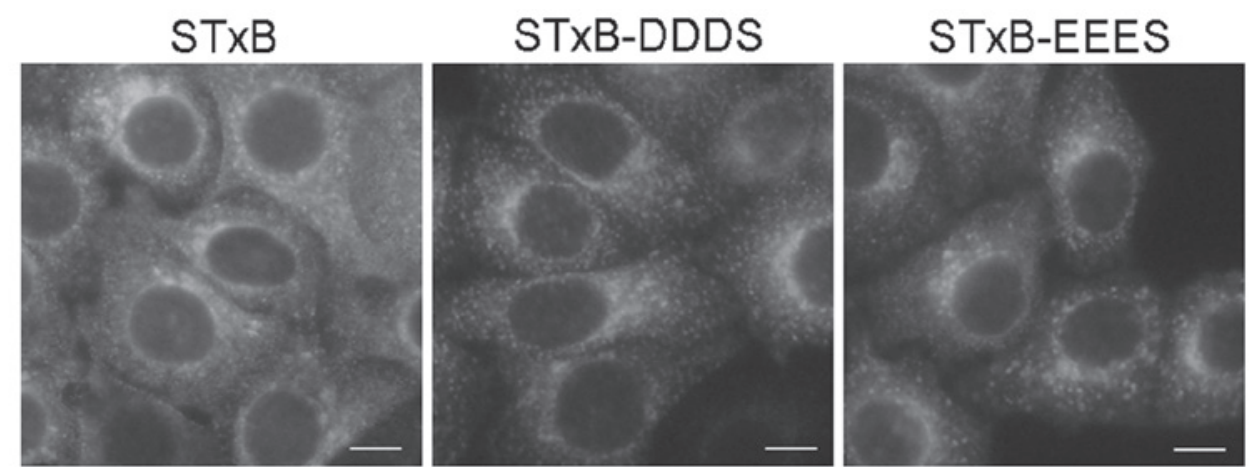

Figure 5. Effects of two types of acidic amino acid tails at the carboxyl-terminus on retrograde transport in HeLa cells. Scale bar, $10 \mu \mathrm{M}$. STxB, Shiga toxin B-subunit. 

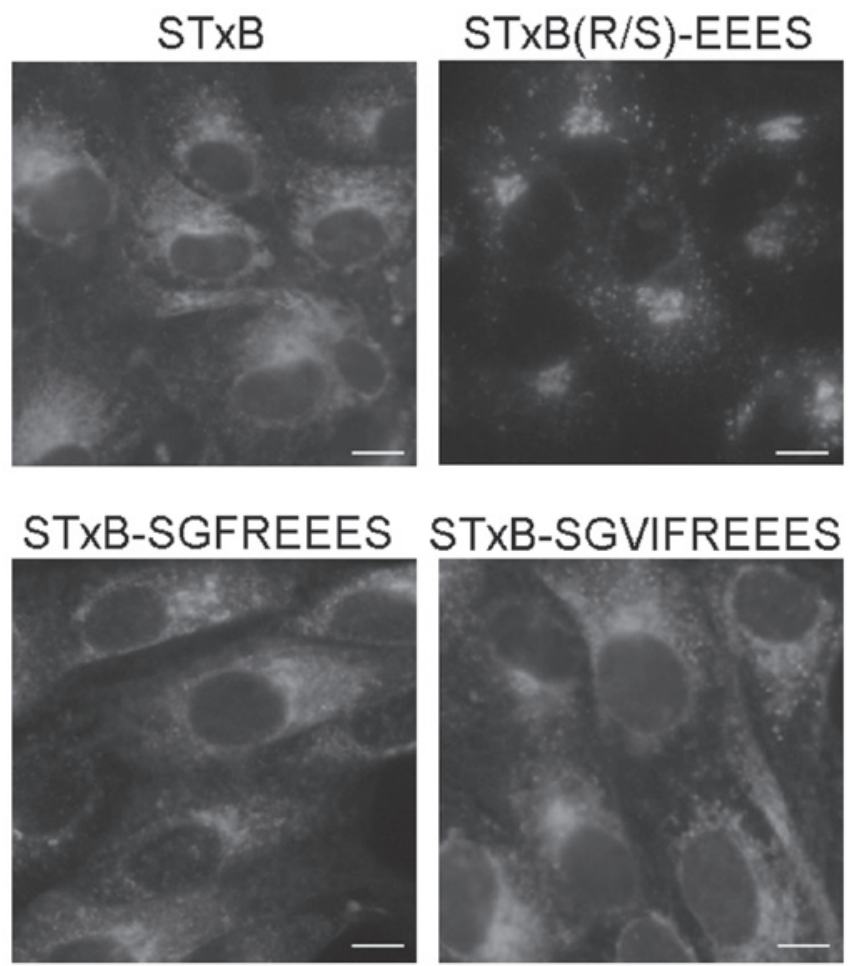

Figure 6. The effects of different positions of the acidic amino acid tail at the carboxyl-terminus of the Shiga toxin B-subunit (STxB) on retrograde transport in HeLa cells. Scale bar, $10 \mu \mathrm{M}$.

evident. However, the accompanying role diminished when the acidic amino acid tail was farther away from the B subunit.

\section{Discussion}

It has been previously reported that STXB, accompanied by its receptor $\mathrm{Gb3}$, can navigate the retrograde pathway from the plasma membrane to the ER via EE/RE and the Golgi apparatus in mammalian cells (25). The C-terminus of the B-fragment was mutated into STxB-Gly-KDEL and STxB-GlyKDELGL, which eventually reached the ER (4), although the effects of the amino acid at the C-terminus on STxB transport remained unclear. This is the first report studying the effect of the C-terminus amino acid of STxB by comparing a series of mutated proteins at the $\mathrm{C}$-terminus on its retrograde transport.

When the end amino acid 'R' was mutated to 'S', STxB (R/S) was transported into the ER from the Golgi at a low speed under the same conditions. It is speculated that the C-terminal basic amino acid ' $R$ ' promotes $S T x B$ retrograde trafficking into the ER and this effect is observed in other neutral amino acids, such as L. However, after adding the acid amino acid E at the end of R, STxB-ES remained in the Golgi, but not after adding the basic and the neutral amino acids. The mechanism for this phenomenon may be that ' $E$ ' shielded the effect of ' $R$ ' by acidic-base neutralization.

Further experiments on the 'ES' tail showed that the length of the tail was essential to its inhibitory activity, and the extent this effect led to STxB-EEEES $\geq$-EEES>-EES>-ES. However, the acidic amino acid tail, 'DDDS', showed a similar effect to 'EEES', indicating that the inhibitory effect was specific to the acidic tail but not to ' $E$ '. When there are internal amino acids between the 'EEES' tail and the C-terminal ' $R$ ', the inhibitory activity decreased, so the 'EEES' tail should be added close to the C-terminus. It was most notable that the inhibitory effect occurred when the C-terminal amino acid 'R' was mutated to ' $S$ ' and then an amino acid tail 'EEES' was added, resulting in $\mathrm{STxB}(\mathrm{R} / \mathrm{S})$-EEES.

Based on the results collected and correlated, we observed and made a hypothesis which explains that there were two factors contributing to this phenomenon. One is a dynamic change, in which the B subunit modifications influenced the transport efficiency on the original target pathway. It may have resulted from changes in the interactions among the five B-subunits or between STxB and Gb3 in the retrograde pathway. Our further experimental findings proved that the concentration of the B-subunit affects the efficiency of their transport (data not shown). The other phenomenon is the change of interaction between the proteins with the environment of the subcellular organelle. An enzyme or signal sequence could interact with the C-terminal of STxB. The acidic amino acid tail affected the circulation of STXB between the Golgi and the ER.

It has been reported that STXB transport is dependent on many regulatory factors, including that of cholesterol content, which affected STxB transport in the direction of the Golgi apparatus (25). The small GTP-binding protein, Rab11 and Rab6, influenced STxB transport from the EE to the Golgi apparatus $(6,26)$. STxB retrograde transport is a complex pathway regulated by many factors and signaling molecules. It has been demonstrated that other toxins such as Cholera toxin and Pseudomonas exotoxin A have a KDEL sequence as an ER retention signal (27). However, STx can also transport into the ER without any signal peptide (20). Therefore, STx may transport into the ER with an unknown signal sequence fragment, which needs to be further investigated.

Gb3 is overexpressed in many human cancers, such as breast cancer, colon carcinoma and lymphoma (28). The STXB retrograde transport pathway has been applied as a delivery tool for chemotherapy against cancer $(13,29)$. Further understanding of the association between the B-subunit structure and its trafficking in cells may contribute to its better development and use. The results from the present study could be used to develop a new method of treatment, not only against STx but also against many types of cancer.

\section{Acknowledgements}

This study was supported by the National Natural Science Foundation of China (No. 30670478), the Doctoral Fund of Ministry of Education of China (20110043120009), the Natural Science Foundation of Jilin Province (201101013) and the Fundamental Research Funds for the Central Universities (10QNJJ012).

\section{References}

1. Sandvig K: Shiga toxins. Toxicon 39: 1629-1635, 2001.

2. Lingwood CA: Role of verotoxin receptors in pathogenesis. Trends Microbiol 4: 147-153, 1996.

3. Merritt EA and Hol WG: AB5 toxins. Curr Opin Struct Biol 5: 165-171, 1995.

4. Johannes L, Tenza D, Antony C and Goud B: Retrograde transport of KDEL-bearing B-fragment of Shiga toxin. J Biol Chem 272: 19554-19561, 1997. 
5. O'Brien AD, Tesh VL, Donohue-Rolfe A, et al: Shiga toxin: biochemistry, genetics, mode of action, and role in pathogenesis Curr Top Microbiol Immunol 180: 65-94, 1992.

6. White J, Johannes L, Mallard F, et al: Rab6 coordinates a novel Golgi to ER retrograde transport pathway in live cells. J Cell Biol 147: 743-760, 1999.

7. Smith RD, Willett R, Kudlyk T, et al: The COG complex, Rab6 and COPI define a novel Golgi retrograde trafficking pathway that is exploited by SubAB toxin. Traffic 10: 1502-1517, 2009.

8. Mallard F, Antony C, Tenza D, Salamero J, Goud B and Johannes L: Direct pathway from early/recycling endosomes to the Golgi apparatus revealed through the study of shiga toxin B-fragment transport. J Cell Biol 143: 973-990, 1998.

9. Kim JH, Johannes L, Goud B, et al: Noninvasive measurement of the $\mathrm{pH}$ of the endoplasmic reticulum at rest and during calcium release. Proc Natl Acad Sci USA 95: 2997-3002, 1998.

10. Marcato P, Griener TP, Mulvey GL and Armstrong GD Recombinant Shiga toxin B-subunit-keyhole limpet hemocyanin conjugate vaccine protects mice from shigatoxemia. Infect Immun 73: 6523-6529, 2005.

11. Kovbasnjuk O, Mourtazina R, Baibakov B, et al: The glycosphingolipid globotriaosylceramide in the metastatic transformation of colon cancer. Proc Natl Acad Sci USA 102: 19087-19092, 2005.

12. Menge C, Stamm I, Wuhrer M, Geyer R, Wieler LH and Baljer G: Globotriaosylceramide $(\mathrm{Gb}(3) / \mathrm{CD} 77)$ is synthesized and surface expressed by bovine lymphocytes upon activation in vitro. Vet Immunol Immunopathol 83: 19-36, 2001.

13. Viel T, Dransart E, Nemati F, et al: In vivo tumor targeting by the B-subunit of shiga toxin. Mol Imaging 7: 239-247, 2008.

14. Poirier C, van Effenterre D, Delord B, Johannes L and Roux D: Specific adsorption of functionalized colloids at the surface of living cells: a quantitative kinetic analysis of the receptormediated binding. Biochim Biophys Acta 1778: 2450-2457, 2008.

15. Janssen KP, Vignjevic D, Boisgard R, et al: In vivo tumor targeting using a novel intestinal pathogen-based delivery approach. Cancer Res 66: 7230-7236, 2006.

16. El Alaoui A, Schmidt F, Amessou M, et al: Shiga toxin-mediated retrograde delivery of a topoisomerase I inhibitor prodrug. Angew Chem Int Ed Engl 46: 6469-6472, 2007.

17. Smith DC, Lord JM, Roberts LM, Tartour E and Johannes L: 1st class ticket to class I: protein toxins as pathfinders for antigen presentation. Traffic 3: 697-704, 2002.
18. Johannes L and Decaudin D: Protein toxins: intracellular trafficking for targeted therapy. Gene Ther 12: 1360-1368, 2005.

19. Bouter A, Delord B, Dransart E, Poirier C, Johannes L and van Effenterre D: Intracellular trafficking of Shiga-toxin-B-subunitfunctionalized spherulites. Biol Cell 100: 717-725, 2008.

20. Sandvig K and van Deurs B: Entry of ricin and Shiga toxin into cells: molecular mechanisms and medical perspectives. EMBO J 19: 5943-5950, 2000.

21. Girod A, Storrie B, Simpson JC, et al: Evidence for a COP-Iindependent transport route from the Golgi complex to the endoplasmic reticulum. Nat Cell Biol 1: 423-430, 1999.

22. Jackson ME, Simpson JC, Girod A, Pepperkok R, Roberts LM and Lord JM: The KDEL retrieval system is exploited by Pseudomonas exotoxin A, but not by Shiga-like toxin-1, during retrograde transport from the Golgi complex to the endoplasmic reticulum. J Cell Sci 112: 467-475, 1999.

23. Hehnly H, Longhini KM, Chen JL and Stamnes M: Retrograde Shiga toxin trafficking is regulated by ARHGAP21 and Cdc42. Mol Biol Cell 20: 4303-4312, 2009.

24. Tai G, Lu L, Johannes L and Hong W: Functional analysis of Arl1 and golgin-97 in endosome-to -TGN transport using recombinant Shiga toxin B fragment. Methods Enzymol 404: 442-453, 2005.

25. Falguieres T, Mallard F, Mallard F, Baron C, Hanau D, Lingwood C, Goud B, Salamero J and Johannes L: Targeting of Shiga toxin B-subunit to retrograde transport route in association with detergent-resistant membranes. Mol Biol Cell 12: 2453-2468, 2001.

26. Miserey-Lenkei S, Waharte F, Boulet A, et al: Rab6-interacting protein 1 links Rab6 and Rab11 function. Traffic 8: 1385-1403, 2007

27. Sandvig K and van Deurs B: Transport of protein toxins into cells: pathways used by ricin, cholera toxin and Shiga toxin. FEBS Lett 529: 49-53, 2002.

28. Falguieres T, Maak M, von Weyhern C, et al: Human colorectal tumors and metastases express Gb3 and can be targeted by an intestinal pathogen-based delivery tool. Mol Cancer Ther 7: 2498-2508, 2008.

29. Johannes L and Romer W: Shiga toxins-from cell biology to biomedical applications. Nat Rev Microbiol 8: 105-116, 2010. 